\title{
ESTUDIO DE LAS COMUNIDADES DE MACROINVERTEBRADOS BENTONICOS Y SU HELMINTOFAUNA EN UN TRAMO DEL RIO OMAÑAS (LEON, NO. DE ESPAÑA)
}

\author{
M.` A. Manzanera Manzanera *; M.^ P. Alvarez Pellitero ** \\ * Laboratorio de Parasitología. Estación Agrícola Experimental de León (CSIC). \\ ${ }^{* *}$ Instituto de Acuicultura Torre de la Sal (CSIC). Ribera de Cabanes (Castellón). \\ Palabras clave: bentic macroinvertebrates, helminthofaune, Omañas River (León).
}

\author{
ABSTRACT \\ STUDY OF THE COMMUNITIES OF BENTHIC MACROINVERTEBRATES AND THEIR \\ HELMINTHOFAUNE IN A STRETCH OF THE OMAÑAS RIVER (LEON, NW SPAIN)
}

In the study of the communities of benthic macroinvertebrates in the stretch of Las Omañas, a predominance of insects -particularly trichoptera and ephemeroptera-, was obsemed in the lotic zones.

The parameters of diversity, richness, frequency and equitability showed certain relation throughout the year, except in June, when the sudden decrease of the diversity while the other parameters remain high, seems to indicate inestability.

Regarding to the helminthological study, only larval Insects and the gastropod Ancylus fluviatilis were found parasitized. Larval trematodes of the families Strigeidae, Sanguinicolidae and Allocreadiidae and larval nematodes (Rhabdochonidae and Mermithidae) were found. The results supply data on the role of Ecdynurus spp. as interniediate hosts of Cystidicoloides tenuissima, and Siphlonurus spp. and Ecdyonurus spp. as second intermediate hosts of Crepidostomum spp. in the stretch studied. Infections were observed in summer and/or autumn, specially in the last period.

\section{INTRODUCCION}

Dada la importancia de las aguas continentales como fuente de recursos naturales, su conocimiento en profundidad, -incluyendo los factores que pueden afectar a su productividad, como son las comunidades parasitarias-, es de sumo interés. En relación con ello, el presente trabajo tiene por objeto el estudio de las comunidades de macroinvertebrados bentónicos en un tramo del río Omaíias, y del papel que algunos de ellos pueden desempeñar como hospedadores intermediarios en los ciclos vitales de distintos helmintos.

\section{MATERIALES Y METODOS}

Los muestreos se realizaron periódicamente, desde junio de 1978 a octubre de 1980, en la estación de

Limnética 3: 133-139 (1987)

(C) Asociación Española de Limnologia, Madrid. Spain
Las Omañas, correspondiente a la zona fluvial de ritrón y situada en la región natural de transición.

Se hicieron muestreos cuantitativos en zonas lóticas, utilizando una "pala de muestreo», similar a la de Macan (1958), en un rectángulo de 30 x $70 \mathrm{~cm}$. Además, se efectuaron algunos muestreos al azar, especialmente en relación con el estudio helmintológico, tanto en las zonas lóticas como en las zonas leníticas de una acequia dependiente del mismo río.

En todos los casos, se registraron los datos físicoquímicos del agua, así como los de profundidad, distancia a la orilla, velocidad de la corriente y temperatura ambiental.

Una vez contados y clasificados los invertebrados de cada muestra, se calcularon los parametros de diversidad (utilizando la fórmula de Shannon \& Weaver, 1949), riqueza, frecuencia y equitabilidad. Asimismo, se calculó la heterogeneidad, según la fórmula de Pielou (citada por Blondel, 1979). 


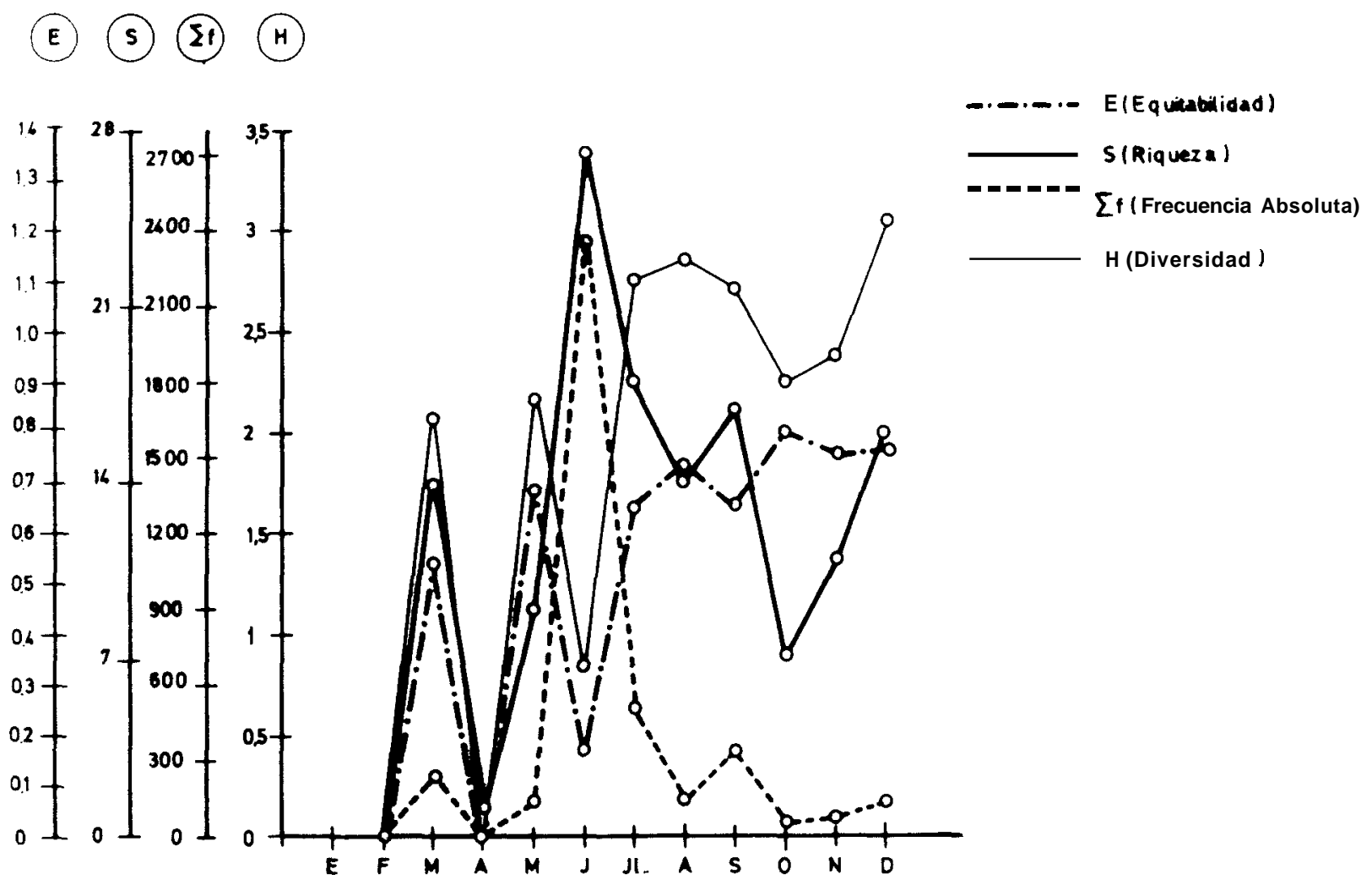

Figura 1.- Representación gráfica de la diversidad y otros parametrosen los distintos meses. Zona lótica. Graphical representation of the diversity and other parameter throughout the year. Lotic area.

El estudio helmintológico se realizó por aplastamiento en placas de triquineloscopia, o mediante disección en algunos casos, de un número representativo de ejemplares de cada especie de macroinvertebrado. En el caso de los moluscos, se observaba previamente la posible emisión de cercarias.

Los helmintos hallados se estudiaron en vivo, siempre que fue posible, aunque también se examinaron algunos ejemplares fijados en alcohol de $70^{\circ}$. Una vez identificados, se calculó la prevalencia de infestación para cada helminto en cada especie de macroinvertebrado.

\section{RESULTADOS}

\section{A) Características físico-químicas de la estación de muestreo}

Los datos correspondientes aparecen en la tabla 1. La temperatura muestra fluctuaciones bastante importantes, con máximos en agosto y septiembre, y una caída bastante brusca en octubre.
La conductividad es, generalmente, débil, y los máximos coinciden con los meses de temperatura más elevada. La concentración de oxígeno disuelto corresponde a la de aguas típicas de salmónidos, Nisbet \& Verneaux (1970).

La alcalinidad total es muy débil, y la dureza total presenta también valores muy bajos, con mayor proporción de $\mathrm{Mg}$ que de $\mathrm{Ca}$, mientras que el contenido en sílice es relativamente elevado.

\section{B) Estudio de las comunidades de macroinvertebra- dos}

Los porcentajes de cada macroinvertebrado recogido en la zona de facies lótica, respecto al total de la muestra, aparecen en la tabla 2.

Los insectos predominan claramente y, dentro de ellos, los tricópteros y los efemerópteros. En cuanto a los tricópteros, muestran la mayor variedad de especies y su frecuencia varía de unos meses a otros, aunque con predominio de una especie de limnefílido y pupas de una especie no determinada. Entre los efemerópteros, predominan claramente los Ecdyonurus 


\begin{tabular}{|c|c|c|c|c|c|c|c|c|c|c|c|}
\hline Mes & Febrero & Marzo & Abril & Mayo & Junio & Julio & Agosto & Septiem. & Octubre & Noviem. & Diciem \\
\hline $\begin{array}{l}\text { V.corr } \\
(\mathrm{m} . / \mathrm{sg})\end{array}$ & 0,60 & 0,42 & 0,75 & 0,33 & 0,75 & 0,45 & 0,03 & 0,05 & 0,01 & 0,32 & 0,25 \\
\hline $\begin{array}{l}\text { Profund. } \\
\text { (om.) }\end{array}$ & 26,00 & 14,50 & 10,00 & 10,00 & 17,50 & 17,00 & 19,00 & 21,00 & 34,00 & 32,00 & 35,00 \\
\hline$\underset{\text { (am.) }}{\text { D.orill. }} 8$ & 830,00 & 155,00 & 150,00 & 225,50 & 150,00 & 150.00 & 155,00 & 150,00 & 115,00 & 75,00 & 240,00 \\
\hline $\begin{array}{c}\text { T.ambien. } \\
\left(\begin{array}{ll}Q & 0\end{array}\right)\end{array}$ & 19,00 & 12,50 & 17,00 & 13,00 & 18,50 & 14,00 & 23,50 & 23,20 & 11,70 & 12,00 & 15,50 \\
\hline 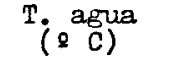 & 2,00 & 7,30 & 11,00 & 11,00 & 14,20 & 14,30 & 19,00 & 17,70 & 8,50 & 6,00 & 6,30 \\
\hline 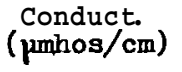 & 26,00 & 30,00 & 34,00 & 38,00 & 44,00 & 50,00 & 55.00 & 55,00 & 42,00 & 30.00 & 36,00 \\
\hline $\begin{array}{r}\text { Oxfgeno } \\
\text { (ppm.) }\end{array}$ & 10,80 & 10,20 & 10,60 & 11,20 & 9,10 & 10,40 & 9,50 & 9,40 & 10,40 & 10,60 & 10,60 \\
\hline $\begin{array}{l}\mathrm{C} \mathrm{O}_{2} \\
(\mathrm{pppm}) \text { ) }\end{array}$ & - & - & - & - & 3,50 & - & 6,00 & 3,00 & 4,00 & - & - \\
\hline $\mathrm{pH}$ & 7,30 & 7,40 & 7,20 & 7,60 & 7,50 & 7,70 & 7,50 & 7,80 & 7,70 & 7,70 & 7,50 \\
\hline $\begin{array}{c}\text { Dureza Ca } \\
(\text { ppm.) }\end{array}$ & 一 & - & - & - & 15,00 & - & 15,00 & 15,00 & 15,00 & - & - \\
\hline$\underset{(\text { ppm. })}{\text { Dureza Mg }}$ & - & 一 & - & 一 & 47,00 & - & 20,00 & 30,00 & 25,00 & - & - \\
\hline $\begin{array}{l}\text { Dure. total } \\
\text { (ppm.) }\end{array}$ & $11-$ & - & - & - & 62,00 & - & 35,00 & 45.00 & 40.00 & - & - \\
\hline $\begin{array}{l}\text { Alcal.to- } \\
\text { tal (ppm.) }\end{array}$ & - & - & - & - & 46,00 & - & 42,00 & 40,00 & 46,00 & - & - \\
\hline $\begin{array}{l}\text { Sflice } \\
(\text { ppm.) }\end{array}$ & - & - & - & - & 7,00 & - & - & 5,00 & 5,00 & - & -- \\
\hline
\end{tabular}

Tabla 1.- Datos físico-químicos.Zona lótica.

Physic-chemical data.

spp., salvo en octubre. Por otra parte, en los muestreos al azar en zonas leniticas se hallaron ejemplares de Siphlonorus spp.

Los moluscos son sumamente escasos, y corresponden a sólo una especie, Ancylus fluviatilis. Sin embargo, en los muestreos al azar en las zonas leniticas se hallaron también algunos ejemplares de los bivalvos Pisidium spp.

La diversidad y los otros tres parámetros analizados (fig. 1), muestran cierta relación a lo largo del año, salvo en el mes de junio, en que hubo un descenso brusco de la diversidad, mientras la riqueza, fre- cuencia y equitabilidad se mantenían elevadas. En cuanto a la heterogeneidad, mostró un valor relativamente bajo $(0,371)$.

\section{C) Estudio helmintológico de los macroinvertebrados}

La helmintofauna hallada en la estación estudiada (tabla 3) incluye trematodos digenéticos y nematodos.

El número de especies de invertebrados que alojaban parásitos fue bajo. Ancylus fluviatilis estaba parasitado por tres digéneos larvarios (metacercarias de 


\begin{tabular}{|c|c|c|c|c|c|c|c|c|c|c|}
\hline Macroinvertebrados Mes & Mar. & Abr. & May. & Jun. & Jul. & Ago. & Sep. & Oct. & Nov. & Dịc. \\
\hline PCRIFERA & 0 & 0 & 0 & 0 & o & 0,62 & o & 0 & o & 0 \\
\hline \multicolumn{11}{|l|}{$\begin{array}{c}\text { PIATYHELMINTHES } \\
\text { Turbellaria }\end{array}$} \\
\hline Dugesia tigrina & 0 & 0 & 0 & 0,04 & 0 & 0 & 0 & 0 & 0 & 0 \\
\hline \multicolumn{11}{|l|}{ ANNELIDA } \\
\hline Lumbricidae & 0 & 0 & 0 & 0 & 0 & 0 & 0,28 & 3,33 & o & 0 \\
\hline Lumbriculidae & 0,74 & 0 & 2,77 & o & 0 & 0 & 0,84 & 0 & 0 & 0 \\
\hline TCTAL & 0,74 & 0 & 2,77 & 0 & 0 & 0 & 1,12 & 3,33 & 0 & 0 \\
\hline \multicolumn{11}{|l|}{ Hirudinea } \\
\hline Erpobdella monostriata & 0 & 0 & 0 & 0,08 & 0,19 & 0 & 1,12 & 0 & 1,14 & 0 \\
\hline Helobdella stapnalis & 0 & 0 & 0 & 0 & 0 & 0 & 0,28 & 3,33 & 0 & 0 \\
\hline Glossiphonia heteroclita & 0 & 0 & 0 & 0,04 & 0 & 0 & 0 & 0 & 0 & 0 \\
\hline TOTAL & 0 & 0 & 0 & 0.12 & 0,19 & 0 & 0 & 3.33 & 1,14 & 0 \\
\hline \multicolumn{11}{|l|}{ MOLLCSCA } \\
\hline \multicolumn{11}{|l|}{ Gastropoda } \\
\hline$\underline{\text { Ancylus fluviatilis }}$ & 0 & 0 & 0 & 0,08 & 0,19 & 1,88 & 0 & 0 & 16,09 & 5,06 \\
\hline \multicolumn{11}{|l|}{ ARTHOPODA } \\
\hline Insecta. Ephemeroptera & & & & & & & & & & \\
\hline Ecdgonurus spp. & 16,79 & 100,00 & 3,47 & 1,70 & 0,352 & 28,30 & 7,32 & 3,33 & 4,59 & 9,49 \\
\hline Baetis spp. & 1,49 & 0 & 2,08 & 0,63 & 0,19 & 0 & 0 & 33,33 & 0 & 5,06 \\
\hline$\underline{\text { Caenis sp. }}$ & 0 & 0 & 0 & 0,04 & 0 & 0 & 0 & 0 & 0 & 0 \\
\hline Procloeon sp. & 0 & 0 & 0 & 0 & 0 & 0 & 0 & 0 & 0 & 1,89 \\
\hline Paralepthophlebia sp. & 0 & 0 & 0 & 0 & 0 & 0,62 & 0 & 0 & 0 & 0 \\
\hline Ephemerella ignita & 0,74 & 0 & 0 & 0,04 & 0,77 & 0,62 & 0,28 & 0 & 0 & 0 \\
\hline Ephemera vulgata & 0 & 0 & 0 & 0 & 0 & 0 & 0,56 & 0 & 0 & 0,53 \\
\hline Heptafenia sp. & 0 & 0 & 0 & 0,29 & O & 0 & 0 & 0 & 0 & 0 \\
\hline TOTAL & 19,02 & 100,00 & 5.55 & 2,72 & 36,45 & 29,55 & 8,16 & 36,66 & 4,59 & 18,98 \\
\hline \multicolumn{11}{|l|}{ Insecta. Plecoptera } \\
\hline Perlidae & 0 & 0 & 1,38 & 0.25 & 1,36 & 9,43 & 17,46 & 0 & $2-29$ & 2,53 \\
\hline Perlodidae & 0,37 & 0 & 3,47 & 0,04 & 0,36 & 2,51 & 0 & 0 & 2,29 & 5,69 \\
\hline Chloroperlidae & 0 & 0 & 0 & 0,04 & 1,16 & 0 & O & 0 & 0 & 0 \\
\hline Taeniopterygidae & 0 & 0 & $c$ & 0 & 0 & 0 & 0 & 0 & 0 & 8,82 \\
\hline Leuctridae & 0 & 0 & 0 & & 0 & 18,86 & 22,53 & 0 & 0 & 0,63 \\
\hline TOTAL & 0,37 & 0 & 4,86 & 0,34 & 2,29 & 30,81 & 40,00 & 0 & 4,59 & 17,08 \\
\hline \multicolumn{11}{|l|}{ Insecta. Coleoptera } \\
\hline Gyrinidae & 1,49 & 0 & 0 & 0,04 & 0 & 0 & 0 & 0 & 0 & 0 \\
\hline Adulto I sin determinar & 0 & 0 & 0 & 0,08 & 0,19 & 0 & 0,28 & 0 & 0 & 0 \\
\hline Adulto $\bar{z}$ sin determinar & 0 & 0 & 0 & 0 & 0,19 & 0 & 0 & 0 & O & 0 \\
\hline TOTAL & 1,49 & 0 & 0 & 0,12 & 0,38 & 0 & 0,28 & 0 & 0 & 0 \\
\hline
\end{tabular}




\begin{tabular}{|c|c|c|c|c|c|c|c|c|c|c|}
\hline Macroinvertebrados & Mar. & Abr. & May. & Jun. & Jul. & Ago. & Sep. & Oct. & Nov. & Dic. \\
\hline \multicolumn{11}{|l|}{ ARTHRCPODA cont. } \\
\hline Insecta. Hemiptera & 0 & 0 & 0 & 0 & 0 & 0 & 0 & 13,33 & 0 & 0 \\
\hline Insecta. Trichoptera & & & & & & & & & & \\
\hline Lepidostomatinae & 0 & 0 & 0 & 0,04 & 0 & 0 & 0 & 0 & 0 & $\mathrm{C}$ \\
\hline Leptoceridae & 0 & 0 & 0 & 0 & 4,48 & 0 & 0 & 0 & 3,44 & 4,43 \\
\hline Goerinae & 0 & 0 & 0 & 0 & 0 & 0,62 & 0 & 0 & 0 & 0 \\
\hline Hydropsychidae & 4,47 & 0 & 0 & 0,93 & 1,36 & 0 & 0 & 0 & 1,14 & 5,69 \\
\hline Sericostomatidae & 0 & 0 & 0 & 0,29 & 0 & 0 & 0 & 0 & 0 & 0 \\
\hline Limnephilidae 1 & $\mathrm{c}$ & 0 & 0 & 0,25 & 0 & 0 & 3,66 & 0 & 0 & 1,89 \\
\hline Limnephilidae 2 & 0 & 0 & 0,69 & 0,12 & 2,29 & 3,14 & 0 & $\mathrm{O}$ & 0 & 0 \\
\hline Limnephilidae 3 & 59,32 & 0 & 43,05 & 2,89 & 6,43 & 22,64 & 31,26 & 0 & 11,49 & 0 \\
\hline Pupas $I$ & 2,98 & 0 & 0 & 0,63 & 0,97 & 0 & 3,38 & 0 & 1,14 & 0 \\
\hline Pupas 2 & 0 & 0 & 0 & 0,08 & 11,89 & 7,54 & 9,85 & 0 & 28,73 & 0 \\
\hline Pupas 3 & 7,08 & $\mathrm{C}$ & 18,05 & 90,13 & 0,24 & 1,84 & 0 & 0 & 0 & 0 \\
\hline TOTAL & 73,88 & 0 & 61,80 & 95,40 & 53,02 & 35,84 & 48,16 & 0 & 45,97 & 12,02 \\
\hline \multicolumn{11}{|l|}{ Insecta. Diptera } \\
\hline$\underline{\text { Dicranota }}$ sp. & $\checkmark, 37$ & 0 & 0 & 0,17 & 0 & 0 & 0 & 0 & 0 & $\mathrm{O}$ \\
\hline Tipula sp. & 0,37 & 0 & 0 & 0,04 & 0 & 0 & 0 & 10,00 & $\mathrm{C}$ & 1,89 \\
\hline Tabanidae & o & 0 & 0 & 0 & 0 & 0 & 0,28 & 0 & 0 & $\mathrm{C}$ \\
\hline Simulidae & $u$ & $u$ & 0 & 0 & 0 & 1,25 & 0 & 0 & 0 & $\mathrm{O}$ \\
\hline Simulidae (pupas) & 2,61 & 0 & 0 & 0 & 0 & 0 & 0 & 0 & 0 & 1,26 \\
\hline Chironomidae & 1,11 & 0 & 25,00 & 0,93 & 6,82 & 0 & 0,28 & 33,33 & 27,58 & 43,67 \\
\hline TOTAL & 44 & 0 & 25,00 & 1,14 & 0 & 1,25 & 0,56 & 43,33 & 27,58 & 46,83 \\
\hline Aracnida. Acarina & 0 & 0 & 0 & 0 & & 0 & 0,28 & 0 & 0 & $\mathrm{C}$ \\
\hline
\end{tabular}

Tabla 2.- Porcentajesde frecuencia de los distintos macroinvertebrados respecto al total de la muestra. Zona lótica. Frequency pertcentage of the differentmacro-invertebrates in relation with the total of the sampling lotic area.

Strigeidae, cercarias de Sanguinicolidae y un esporocisto no determinado), pero siempre con prevalencias muy bajas.

El resto de los invertebrados parasitados eran insectos. Ecdyonurus spp. alojaban metacercarias de Crepidostomum spp. y larvas III de Cystidicoloides tenuissima. En cuanto a Siphlonurus spp., sólo estaban parasitadas por metacercarias de Crepidostomum spp., pero con elevada prevalencia (60\%). Por fin, una larva de Chironomidae alojaba una larva de mermítido.

Por lo que se refiere a la estacionalidad, en general se hallaron infestaciones en verano $y / u$ otoño, aunque mas frecuentemente y con mayor prevalencia en este último periodo, único en que se encontró Sanguinicola sp. y Metacercaria sp. (Strigeidae).

\section{DISCUSION}

El hecho de que la alcalinidad total sea débil, podría indicar una productividad relativamente baja, se- gún los criterios de Nisbet \& Verneaux (1970). Por otra parte, la baja proporción de calcio y el contenido relativamente elevado en sílice, podrían estar en relación con la escasez de caliza y el predominio de cuarcitas silíceas en la zona.

En cuanto a las comunidades de macroinvertebrados, el predominio de insectos en las zonas Ióticas, ha sido observado también en otros ríos de León (González Lanza, co. pers.; Torres Hevia, com. pers.). Por otra parte, la baja frecuencia de moluscos puede estar relacionada con la escasez de calizas. En general, las especies de macroinvertebrados determinadas y sus frecuencias, indican zonas no contaminadas, lo que concuerda también con sus características físicoquímicas. Sin embargo, en los meses de febrero y abril, la diversidad y riqueza faunistica indican que la comunidad se halla fuertemente impactada, debido probablemente al aumento de caudal del río.

Por lo que se refiere a la helmintofauna hallada, es relativamente pobre en especies y sus prevalencias son, generalmente, bajas. En cuanto a otros estudios helmintológicos de macroinvertebrados bentónicos, 


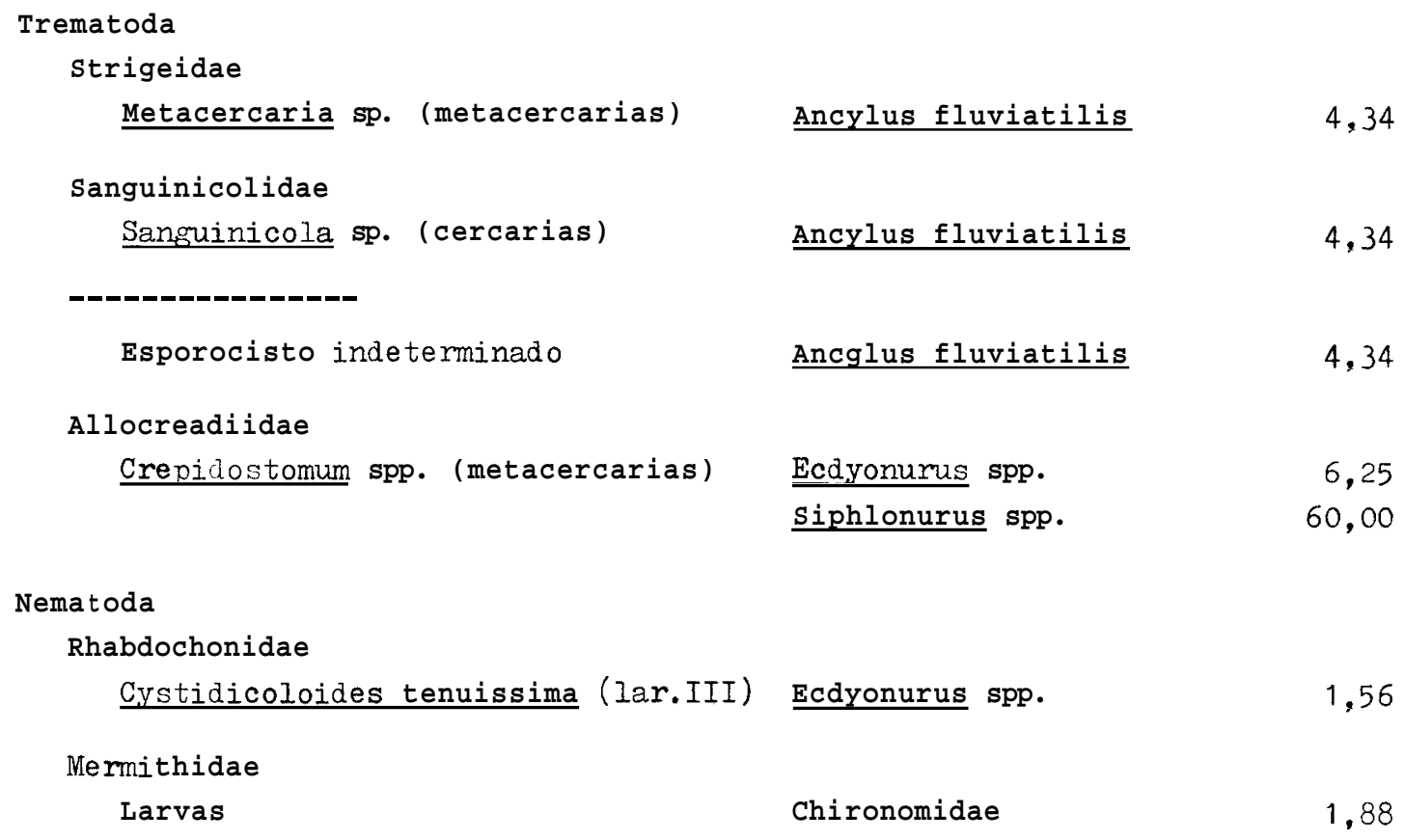

\section{Nema toda}

Rhabdochonidae

Cystidicoloides tenuissima (Iar.III)

Ecdyonurus spp.

Tabla 3.- Helmintos hallados y prevalenciade las infestacionesen los correspondientes hospedadores Helminths found and frequency of the infestationsin their corresponding hosts.

en general son escasos, y se refieren a moluscos en la mayoría de los casos, Simón Vicente (1979) y Simón Martín (1983) hallaron también cercarias de Sanguinicola sp. en Ancylus fluviatilis de Salamanca, pero con prevalencia algo mayor. Por lo que se refiere a los insectos, Ilyushina (1975) obtuvo resultados en parte diferentes a los nuestros, ya que los odonatos y megalópteros eran los órdenes parasitados por mayor número de especies, seguido de los efemerópteros y dípteros.

Los hospedadores definitivos de algunos de los helmintos hallados, Crepidostomum spp. y Cystidicoloides tenuissima, son las truchas comunes, según estudios realizados previamente en el mismo río (Alvarez Pellitero, 1979). Los resultados del presente trabajo demuestran que las ninfas de Ecdyonurus spp. pueden actuar como hospedadores intermediarios de Cystidicoloides tenuissima en este río. Otros autores atribuyen este papel a distintos efemerópteros, pero no a las Ecdyonurus spp. (Moravec, 1971; Moravec \& De, 1982). Por otra parte, los segundos hospedadores intermediarios de Crepidostomum spp., según nuestro estudio, son las ninfas de Siphlonurus spp. y
Ecdyonurus spp. Especies de Siphlonurus han sido señaladas como hospedadores de estos trematodos por algunos autores (Klein, 1967; Bwathondi, 1976), pero Ecdyonurus sp. sólo ha sido indicada por Awachie (1968) alojando metacercarias muertas.

Por lo que se refiere a la estacionalidad, la existencia de dos periodos principales de infestación ha sido señalada también para otros macroinvertebrados (Natsvlishvili, 1973; Simón Vicente, 1979), aunque Pike (1968) indica una situación variable para las infestaciones por metacercarias. También se ha indicado una estacionalidad bimodal para los adultos de algunos de los helmintos hallados, como Crepidostomum spp. y Cystidicoloides tenuissirna, en sus hospedadores definitivos, las truchas, del mismo hábitat (Alvarez Pellitero, 1979).

\section{AGRADECIMIENTOS}

Agradecemos al Prof. Estanislao Luis Calabuig sus sugerencias y colaboración en la elaboración del analisis de las comunidades de macroinvertebrados. 


\section{BIBLIOGRAFIA}

Alvarez Pellitero, M.a P., 1979. Helmintocenosis del tracto digestivo de la trucha de rio en los rios de León. Institución Fray Bemardino de Sahagún. Excma. Diputación Provincial (CSIC), León.

Awachie, J.B.E. 1968. On the bionomics of Crepidostomum metoecus (Braum, 1900) and Crepidostomum farionis (Müller, 1784) (Trematoda: Allocreadiidae). Parasitology, 58: 307-324.

Blondel, J., 1979. Biogéographie et Ecólogie. Masson et Cie., París.

Bwathondi, P.O.J., 1976. Infection of trout, Salmo trutta L. by Crepidostomum metoecus (Braum, 1900) in Loch of Strathberg, NE Scotland. Purasitology, 73: X.

Ilyushina, T.L., 1975. Papel de los insectos acuáticos en los ciclos vitales de los trematodos. Trudy Biol. Inst. Sib. Otdel. Akad. Nauk SSSR, 17: 53-94 (en ruso).

Klein, W.D., 1967. Crepidostomum farionis in rainbow trout. M.S. Thesis. Colorado State University, Ft., Collins. Colorado.

Macan, T.T., 1958. Methods of sampling the bottom fauna in stony streams. Mitt. Int. Ver. Limnol., 8: 1-21.

Moravec, F., 1971. On the life history of the nematode Cysti- dicoloides tenuissima (Zeder, 1800) in the River Bystrice, Czechoslovakia. Folia parasit, Praha, 18: 107-112.

Moravec, F. \& De, N.C., 1982. Some new data on the bionomics of Cystidicoloides tenuissima (Nematoda: Cystidicolidae). Vest. csl. Spol. Zool., 46: 100-108.

Natslishvili, M.G., 1973. Dinámica estaciona1 de las infestaciones por trematodos larvarios en moluscos del área de Tbilisi. Soobsh. Akad. Nauk. Grudz. SSR, 70: 725-727 (en ruso). Nishet, M. \& Verneaux, J., 1970. Composantes chimiques des eaux courantes. Discussion et proposition de classes en tant que basses d'interprêtation des analyses chimiques. Annls. Limnol., 6: 161-190.

Pike, A.W., 1968. The distribution and incidence of larval trematodes in the freshwater fauna of the Wentloog level. South Wales. J. Zool., 155:293-309.

Shannon, C.E. \& Weaver, W., 1949.The Mathematical Theory of Communication. University of Illinois Press, Urbana.

Simón Martín, F., 1983. Sobre la ecologia y ciclo biológico de Sanguinicola sp. (Trematoda, Sanguinicolidae) en ciprinidos de la provincia de Salamanca. Serie: Resúmenes de Tesis Doctorales. Facultad de Biología. Universidad de Salamanca. Simón Vicente, F., 1979. Trematodos larvarios y sus moluscos hospedadores en Salamanca. Revista Ibérica de Parasitologia, 39: 241-250. 